\title{
Different phenotypes in identical twins with Cerebrotendinous Xanthomatosis: case series
}

Authors: Dénes Zádori', László Szpisjak, László Madar², Viktória Evelin Varga ${ }^{3}$, Bernadett Csányi ${ }^{4}$, Krisztina Bencsik ${ }^{1}$, István Balogh ${ }^{2}$, Mariann Harangi ${ }^{3}$, Éva Kereszty $^{4}$, László Vécsei ${ }^{1,5}{ }^{*}$, Péter Klivényi ${ }^{1}$

\section{Affiliations:}

${ }^{1}$ Department of Neurology, Faculty of Medicine, Albert Szent-Györgyi Clinical Centre, University of Szeged, Szeged, Hungary

${ }^{2}$ Division of Clinical Genetics, Department of Laboratory Medicine, University of Debrecen, Debrecen, Hungary

${ }^{3}$ Division of Metabolic Diseases, Department of Internal Medicine, University of Debrecen, Debrecen, Hungary

${ }^{4}$ Department of Forensic Medicine, Faculty of Medicine, Albert Szent-Györgyi Clinical Centre, University of Szeged, Szeged, Hungary

${ }^{5}$ MTA-SZTE Neuroscience Research Group, Szeged, Hungary

*Corresponding author:

LászlóVécsei, MD, PhD, DSc

Department of Neurology, Faculty of Medicine, Albert Szent-Györgyi Clinical Centre, University of Szeged,

Semmelweis u. 6, H-6725 Szeged, Hungary

Phone: +36 62 545351; 
Fax: +3662545597

E-mail: laszlo.vecsei@med.u-szeged.hu

\section{Acknowledgement}

This study was funded by Hungarian Brain Research Program - Grant No. KTIA_NAP_13A_II/18. Dénes Zádori was supported by the János Bolyai Research Scholarship of the Hungarian Academy of Sciences. The linguistic corrections were made by a native English reader, Jennifer Tusz from Canada.

\section{Conflict of interest}

The authors declare that they have no conflict of interest. 


\begin{abstract}
Cerebrotendinous Xanthomatosis (CTX) is a rare genetically determined error of metabolism. The characteristic clinical symptoms are diarrhea, juvenile cataracts, tendon xanthomas and neuropsychiatric alterations. The aim of this study is to present a pair of identical adult twins with considerable differences in the severity of phenotype. With regards to neuropsychiatric symptoms, the predominant features were severe Parkinsonism and moderate cognitive dysfunctions in the more affected individual, whereas these alterations in the less affected patient were only very mild and mild, respectively. The characteristic increase in the concentrations of serum cholestanol and the lesion volumes in dentate nuclei in the brain assessed with magnetic resonance imaging were quite similar in both cases. The lifestyle conditions, including eating habits of the twin pair, were quite similar as well, therefore currently unknown genetic modifiers or certain epigenetic factors may be responsible for the differences in severity of phenotype. This case series serves as the first description of an identical twin pair with CTX presenting heterogeneous clinical features.
\end{abstract}

Keywords: Cerebrotendinous Xanthomatosis, Parkinsonism, cognitive dysfunction, identical twins, heterogeneous phenotype 


\section{Introduction}

Cerebrotendinous Xanthomatosis (CTX) is an autosomal recessively inherited condition belonging to the group of inborn errors of metabolism [1]. It affects approximately 1 to 2 out of 100,000 individuals. The pathogenic mutations are located in the CYP27A1 gene mapped to $2 q 35$ [2,3]. The gene product, sterol 27-hydroxylase, which is expressed in the central nervous system, liver, lungs, duodenum and endothelial cells, takes part in the appropriate production of bile acids from cholesterol [4]. Sterol 27-hydroxylase deficiency results in the accumulation of $7 \alpha$-hydroxy-4-cholesten-3-one and its metabolites, including cholestanol, and also results in the insufficient production of chenodeoxycholic acid [1]. The main characteristic signs and symptoms of CTX are diarrhea, juvenile cataracts, tendon xanthomas and neuropsychiatric alterations, including cognitive and psychiatric disturbances, pyramidal and/or cerebellar signs, seizures and Parkinsonism [3]. Although there is a marked heterogeneity of signs and symptoms in CTX patients, even in intrafamilial cases [2], no phenotypic variability has yet been reported in twins or triplets, [5]. The aim of the current study is to present the considerably different phenotypes of a pair of identical adult twins diagnosed with CTX.

\section{Case reports}

One member of a 40-year-old female twin pair was first admitted to our neurology department in 2016 with the aim of a diagnostic work-up on her movement disorder. She had already had cataracts and glaucoma in childhood, but her movement and speech only began to worsen progressively from 2013. Additionally, she complained of an episode of pronounced diarrhea and gastrointestinal discomfort in 2015 , and her parents mentioned memory problems, anxiety and impatience as well. On neurological examination, she presented signs of a movement disorder with dominating Parkinsonism (moderate symmetric hypo- and bradykinesia, rigor, 
mainly right-sided occasional limb rest tremor, severe postural instability, freezing of gait, antecollis, hypomimia, severe dysarthria and mild seborrhea; Movement Disorder Society Unified Parkinson's Disease Rating Scale (MDS-UPDRS) part III: 75 points in OFF state; Suppl. video 1) showing some levodopa response (MDS-UPDRS part III: 56 points in ON state, i.e., $25.3 \%$ improvement; Suppl. video 2). Furthermore, the patient had mild cerebellar ataxia and pyramidal signs (brisk patellar reflex and ankle clonus on the right side with bilateral Babinski sign) as well. The unaided stance and gait could not be implemented. The neuropsychological assessment demonstrated moderate cognitive dysfunctions in light of 65/100 points in Addenbrooke's Cognitive Examination (ACE) and 24/30 points in the MiniMental State Examination (MMSE). The skull MRI revealed T2 and FLAIR abnormal signals in the dentate nuclei and some supratentorial white matter alterations (Figures 1A, B, C and D). The clinical picture raised the possibility of CTX which was supported by elevated serum cholestanol levels (31 $\mu \mathrm{M}$; normal range: 2-12.6 $\mu \mathrm{M})$. The genetic testing for a diseasecausing mutation in the CYP27A1 gene revealed a known pathogenic homozygous frameshift mutation in exon 4 (c.819delT, p.D273EfsTer13).

With regards to family history, the other member of the twin pair also had juvenile cataracts and glaucoma and was suspected to have some kind of immunological disorder. Furthermore, her parents also reported less expressed memory problems, anxiety and impatience.

Otherwise, the family history of the assessed patient was irrelevant. Despite the absence of pronounced neurological features, this second patient was also screened for possible signs of CTX. The examination revealed signs of slight Parkinsonism (mainly left-sided slight hypoand bradykinesia and rigor on provocation in the left upper limb, and moderate postural instability; MDS-UPDRS part III: 8 points in OFF state; Suppl. video 3) and mild sensory ataxia with a slightly broad-based gait. With regards to cognitive function, the neuropsychological assessment demonstrated mild alterations in light of 72/100 points in 
ACE and 27/30 points in the MMSE. The abnormal signals in the dentate nuclei were present as well (Figures 1E and F). Furthermore, the MRI revealed asymptomatic mega cisterna magna (Figures 1E, F and $\mathrm{H}$ ) and a small tentorial meningeoma (Figures $1 \mathrm{G}$ and $\mathrm{H}$ ). Despite the less expressed clinical alterations when compared to her twin sister, similarly elevated serum cholestanol levels were detected $(36.8 \mu \mathrm{M})$. The genetic testing identified the same disease-causing mutation in the CYP27Al gene.

The considerably different phenotypes raised the question of whether the twin pair is identical or not. The following 15 short tandem repeat markers were analyzed for that purpose:

D8S1179, D21S11, D7S820, CSF1PO, D3S1358, TC11, D13S317, D16S539, D2S1338, D19S433, VWA, TPOX, D18S51, D5S818 and FGA. The DNA profile of the 2 subjects was completely identical, so they are confirmed to be identical twins. The parents were heterozygous for the assessed mutation and they did not show any sign or symptom of CTX.

\section{Discussion}

Although the role of CYP27A1 gene mutations in the pathogenesis of CTX is wellestablished, no genotype-phenotype correlation can be determined. When compared with previously reported CTX patients, the clinical features of our identical twin pair were slightly different from typical cases (including those with the same pathogenic mutation [6]), lacking tendon xanthomas and seizures, and presenting a predominant Parkinsonian syndrome, especially in the more severely affected patient. However, the Parkinsonian features were similar to that of a previously published case [7]. Nevertheless, the major aim of this case series is not only the simple demonstration of signs and symptoms in this identical twin pair, but to draw attention to the considerable differences in the severity of their clinical features and the identification of possible underlying factors. Biochemical testing revealed similar alterations in cholestanol concentrations, but it is known that the levels of this metabolite have 
no correlation with clinical phenomena [8]. Although the role of environmental factors has been suggested to be possibly responsible for the clinical differences [2], the fact that our twin pair has been continuously living together with their parents and their eating habits are similar as well, makes this theory questionable. However, currently unknown genetic modifiers of certain epigenetic factors may play a role in clinical heterogeneity. The clarification needs further studies.

In conclusion this case series study serves as the first report of identical twins diagnosed with CTX demonstrating remarkably different phenotypes.

\section{Ethical approval}

Written informed consent was obtained from the patients for video recording and the publication of this study (institutional research committee registration numbers are 150/2014. and 44/2016., respectively). All procedures applied during the assessment of patients were in accordance with the ethical standards of the institutional research committee and with the 1964 Helsinki declaration and its later amendments or comparable ethical standards.

\section{References}

1. Bjorkhem I (2013) Cerebrotendinous xanthomatosis. Curr Opin Lipidol 24 (4):283-287. doi:10.1097/MOL.0b013e328362df13

2. Verrips A, Hoefsloot LH, Steenbergen GC, Theelen JP, Wevers RA, Gabreels FJ, van Engelen BG, van den Heuvel LP (2000) Clinical and molecular genetic characteristics of patients with cerebrotendinous xanthomatosis. Brain 123 ( Pt 5):908-919. doi:10.1093/brain/123.5.908

3. Gallus GN, Dotti MT, Federico A (2006) Clinical and molecular diagnosis of cerebrotendinous xanthomatosis with a review of the mutations in the CYP27A1 gene. Neurol 
Sci 27 (2):143-149. doi:10.1007/s10072-006-0618-7

4. Chiang JY (1998) Regulation of bile acid synthesis. Front Biosci 3:d176-193.

5. Nagai Y, Hirano M, Mori T, Takakura Y, Tamai S, Ueno S (1996) Japanese triplets with cerebrotendinous xanthomatosis are homozygous for a mutant gene coding for the sterol 27hydroxylase (Arg441Trp). Neurology 46 (2):571-574. doi:10.1212/WNL.46.2.571

6. Leitersdorf E, Reshef A, Meiner V, Levitzki R, Schwartz SP, Dann EJ, Berkman N, Cali JJ, Klapholz L, Berginer VM (1993) Frameshift and splice-junction mutations in the sterol 27hydroxylase gene cause cerebrotendinous xanthomatosis in Jews or Moroccan origin. J Clin Invest 91 (6):2488-2496. doi:10.1172/JCI116484

7. Dotti MT, Federico A, Garuti R, Calandra S (2000) Cerebrotendinous xanthomatosis with predominant parkinsonian syndrome: further confirmation of the clinical heterogeneity. Mov Disord 15 (5):1017-1019. doi:10.1002/1531-8257(200009)15:5<1017::AID-MDS1043>3.0.CO;2-F 8. Mignarri A, Rossi S, Ballerini M, Gallus GN, Del Puppo M, Galluzzi P, Federico A, Dotti MT (2011) Clinical relevance and neurophysiological correlates of spasticity in cerebrotendinous xanthomatosis. J Neurol 258 (5):783-790. doi:10.1007/s00415-010-5829-4

\section{Figure Legend}

Figure 1. The main characteristic magnetic resonance imaging (1.5 Tesla) features of our patients with Cerebrotendinous Xanthomatosis. The lesion of the dentate nuclei is prominent in the more affected member (black arrows; T2-wheighted images: A; FLAIR images: B) and the less affected member (black arrows; T2-wheighted images: E; FLAIR images: F) of the identical twin pair. Furthermore, the more affected member demonstrated supratentorial white matter alterations (black arrows; T2-wheighted images: C; FLAIR images: D). In the less affected member the MRI revealed asymptomatic mega cisterna magna (grey arrows; axial 
T2-wheighted images: E; axial FLAIR images: F; coronal T1-wheighted images: H) and a small contrast-enhancing tentorial meningeoma (black arrows; sagittal T1-wheighted images: G; coronal T1-wheighted images: H)

\section{Video legends for supplementary materials}

Supplementary video 1. The Parkinsonian features of the more affected member of the identical twin pair in OFF state.

Supplementary video 2. The Parkinsonian features of the more affected member of the identical twin pair in $\mathrm{ON}$ state following levodopa challenge.

Supplementary video 3. The very slight Parkinsonian features of the less affected member of the identical twin pair. 

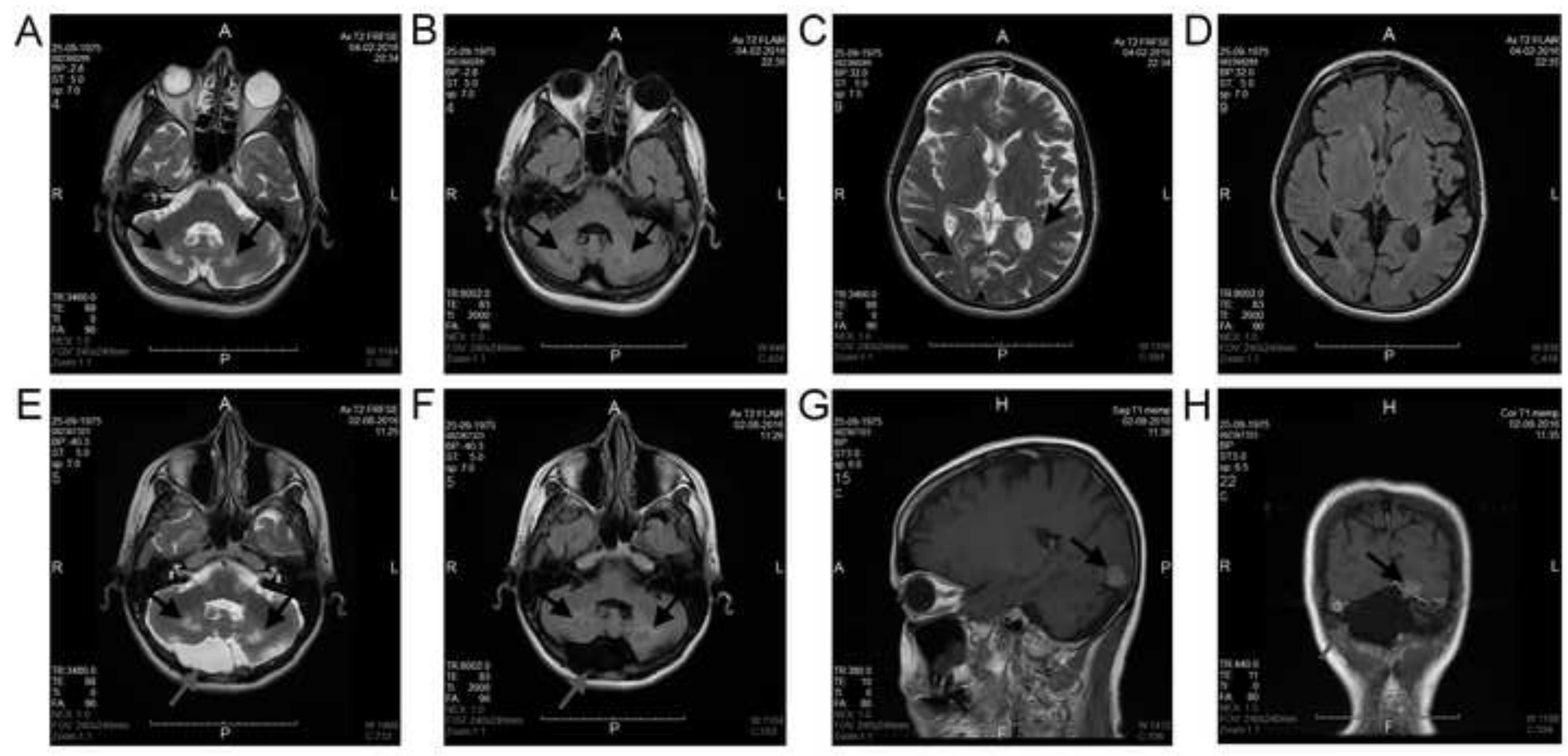